\title{
Genetic predictors of response to specific drugs in primary myelofibrosis
}

\author{
Domenico Penna', Natasha Szuber', Terra L. Lasho', Christy M. Finke', Rangit R. Vallapureddy ${ }^{1}$, Curtis A. Hanson², \\ Rhett P. Ketterling ${ }^{3}$, Animesh Pardanani ${ }^{1}$, Naseema Gangat ${ }^{1}$ and Ayalew Tefferi ${ }^{1}$
}

Primary myelofibrosis (PMF) constitutes clonal expansion of myeloid cells and is characterized by "driver" (i.e., $J A K 2, C A L R$, and MPL) and other mutations or DNA variants, such as $A S X L 1, T E T 2, S R S F 2, I D H 1, I D H 2$, and $U 2 A F 1^{1-3}$. Patients with PMF have an estimated median survival of 6 years and causes of death include leukemic transformation; in addition, quality of life is markedly impaired in PMF as result of frequent red blood cell transfusion requirement, markedly enlarged spleen and severe constitutional symptoms ${ }^{4,5}$. At present allogeneic stem cell transplant (ASCT) is the only treatment modality with potential to either cure the disease or prolong survival of patients with PMF, while current drug therapy offers palliative value only (e.g., reduction in spleen size, reduction of constitutional symptoms, and improvement in anemia $)^{6,7}$. In this regard, hydroxyurea (HU), JAK2 inhibitors, and interferon (IFN)- $\alpha$ are often used for symptomatic splenomegaly: JAK2 inhibitors for constitutional symptoms and immunomodulatory drugs (IMiDs), erythropoiesis-stimulating agents (ESAs), and androgens for anemia ${ }^{8}$.

The current study was approved by the Mayo Clinic Institutional Review Board (IRB) and represents a retrospective evaluation of specific drug response, in terms of anemia or splenomegaly, in patients with PMF receiving these agents as first-line treatment. Diagnoses and treatment approaches were in accordance with what was considered standard of care at the time of initial diagnosis or first referral ${ }^{9}$. Study patients were recruited from the Mayo Clinic, Rochester, MN, USA. Diagnoses were

Correspondence: Ayalew Tefferi (tefferi.ayalew@mayo.edu)

${ }^{1}$ Divisions of Hematology, Departments of Internal Medicine and Laboratory Medicine, Mayo Clinic, Rochester, MN, USA

${ }^{2}$ Divisions of Hematopathology, Departments of Internal Medicine and Laboratory Medicine, Mayo Clinic, Rochester, MN, USA

Full list of author information is available at the end of the article. according to the 2016 World Health Organization criteria ${ }^{10}$. To ascertain the role of genetic biomarkers, study inclusion criteria included availability of karyotype and next-generation sequencing (NGS)-derived mutation information.

Variables evaluated included those that are currently listed in MIPSS70 (mutation-enhanced international prognostic scoring system for transplant-age patients), MIPSS70 + version 2.0 (karyotype-enhanced MIPSS70), and GIPSS (genetically-inspired prognostic scoring system $)^{11-13}$. For the purposes of the current study, conventional response criteria were modified to reflect clinical benefit assessment without strict adherence to criteria designed for clinical trials. Accordingly, spleen response was evaluated only in patients with palpable splenomegaly and was defined as a minimum $50 \%$ reduction in palpable spleen size, regardless of response duration. Anemia response was evaluated only in patients with hemoglobin level $<10 \mathrm{~g} / \mathrm{dl}$ and was defined as achieving transfusion-independence lasting for at least 1 month or an increase in hemoglobin of $2 \mathrm{~g} / \mathrm{dl}$, regardless of response duration ${ }^{14}$. Statistical analyses considered clinical and laboratory parameters obtained at time of diagnosis or first referral, which coincided, in all instances, with time of sample collection for mutation analysis. Conventional methods were used for statistical analysis (JMP ${ }^{\circledR}$ Pro 13.0.0 software; SAS Institute, Cary, NC).

A total of 432 cytogenetically- and molecularlyannotated patients with PMF were accessed (Supplementary Table 1 ) in order to identify 333 patients who received first-line treatment with $\mathrm{HU}(n=97)$, JAK2 inhibitors $(n=41)$, IFN- $\alpha \quad(n=22)$, IMiDs $(n=58)$, androgens $(n=19)$, ESAs $(n=54)$, and various other drugs $(n=42)$, and were evaluable for response. Presenting features of the 333 study patients (median age 64 years; $68 \%$ males) included palpable splenomegaly (77\%), 


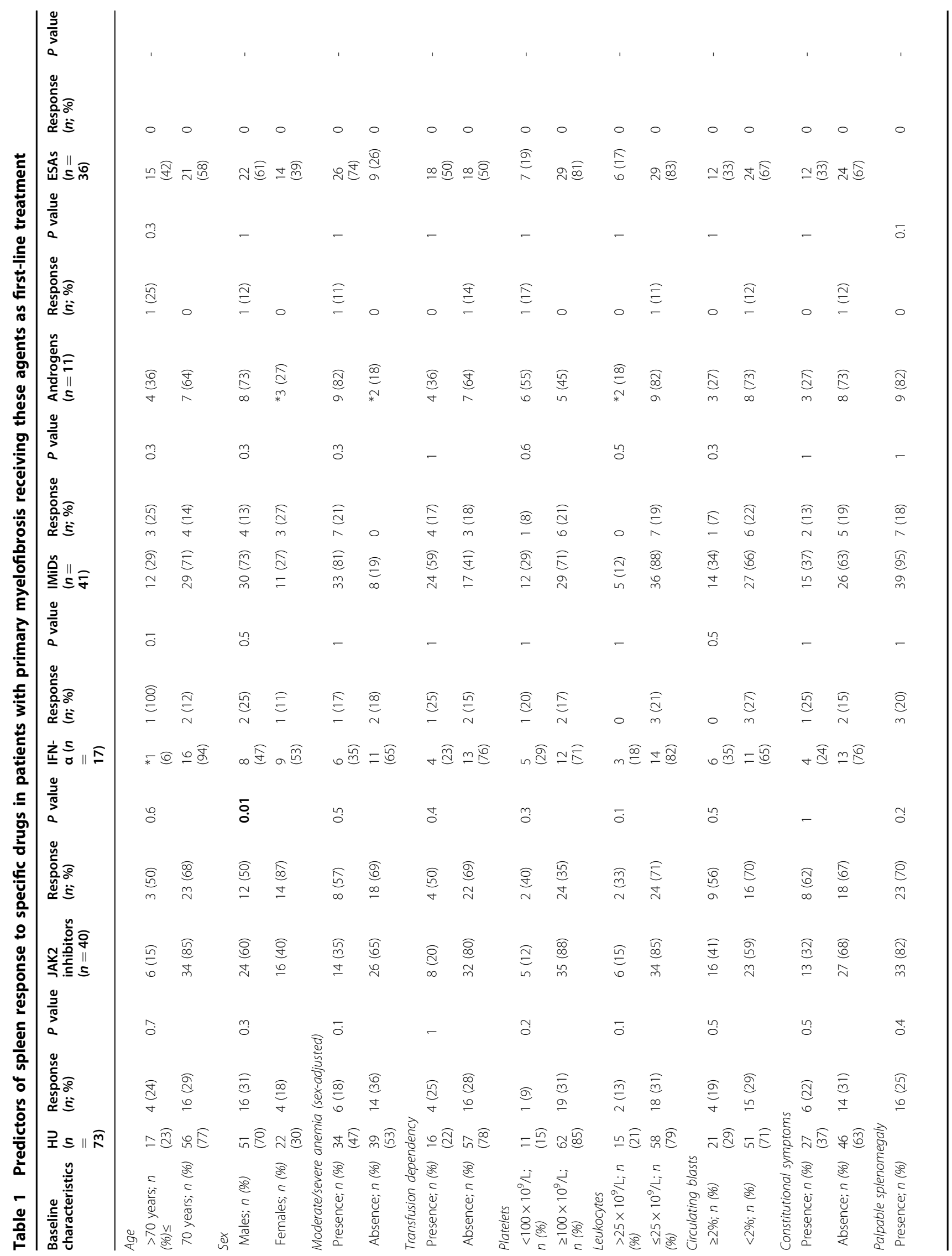




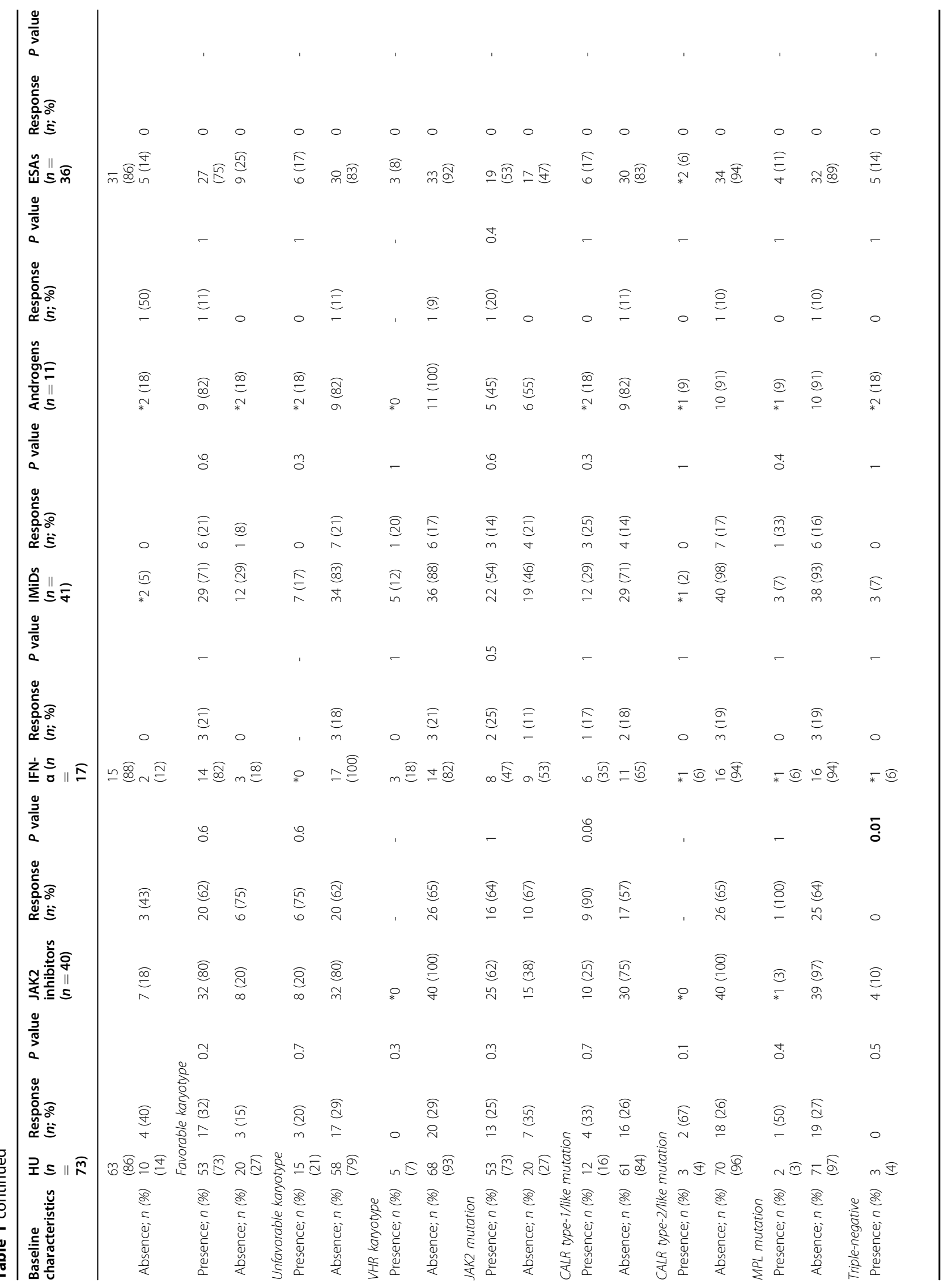




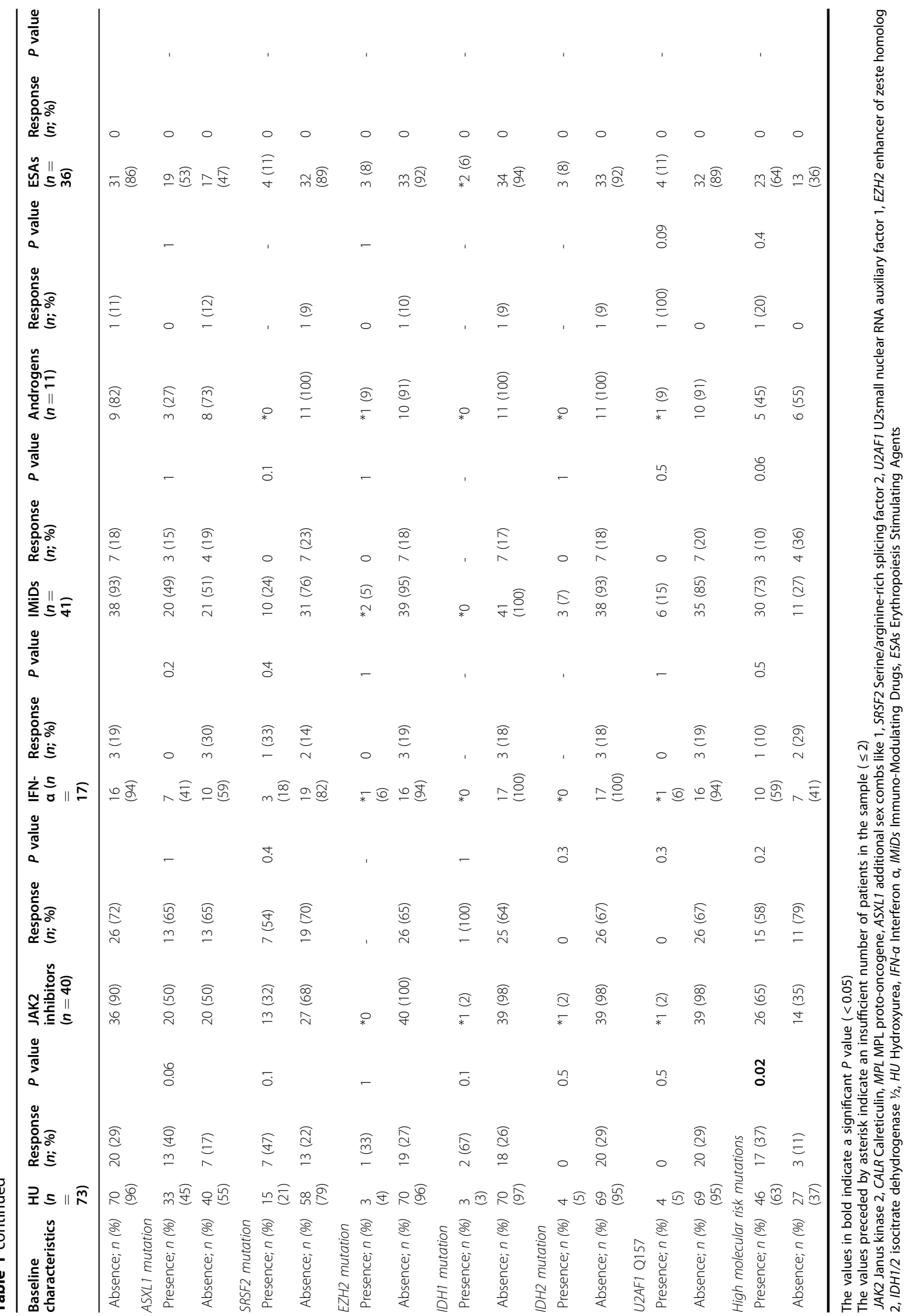




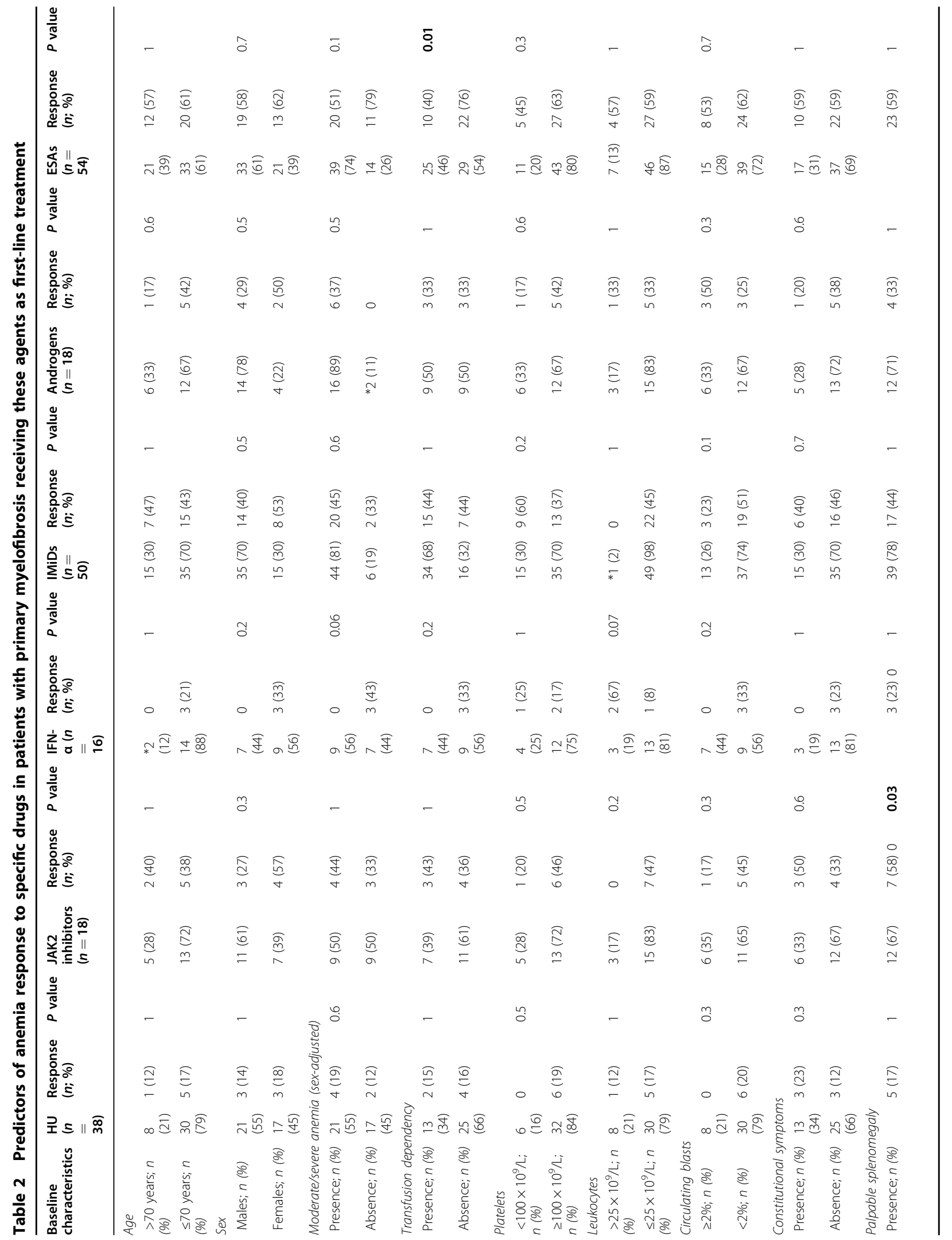




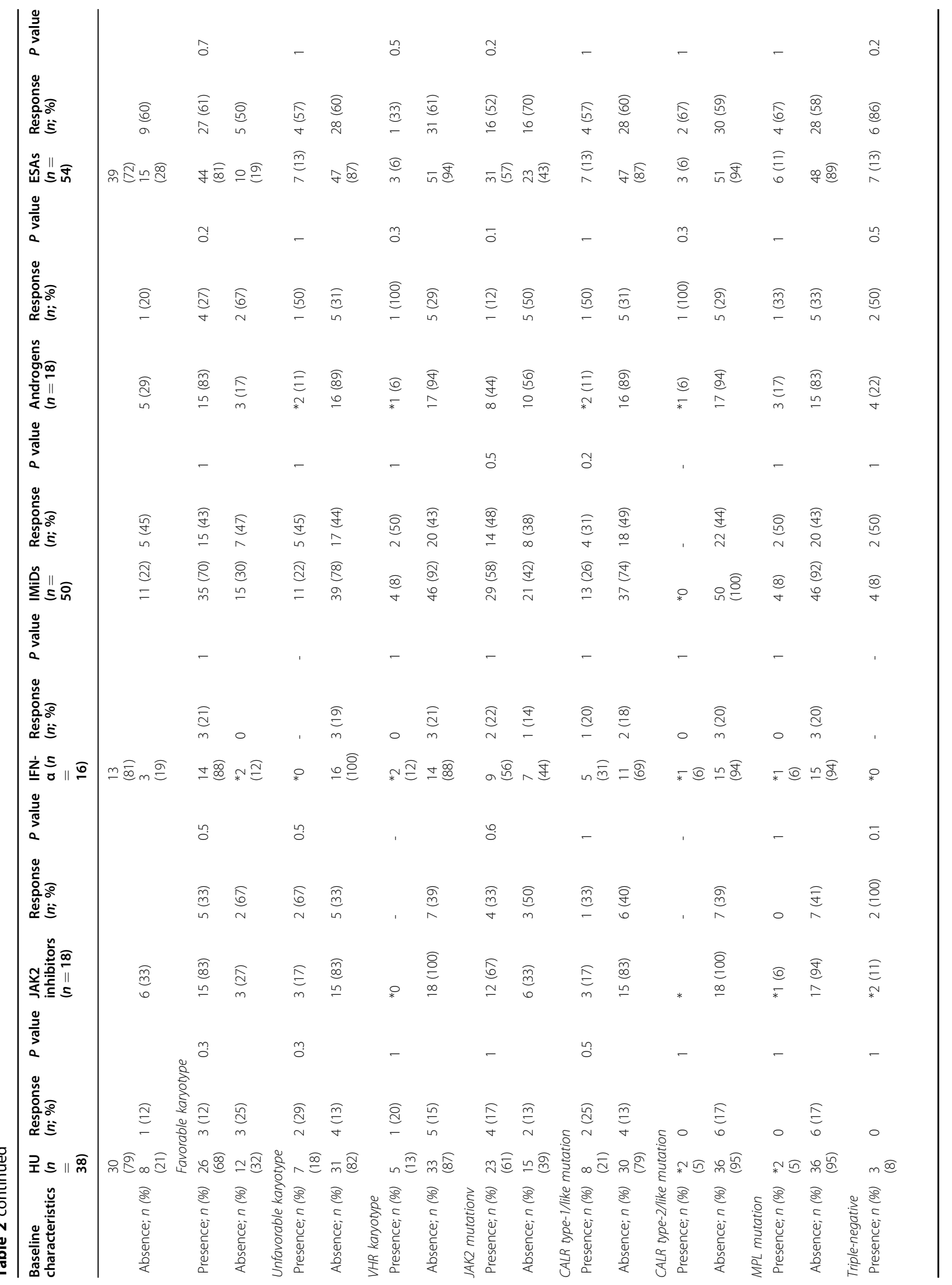




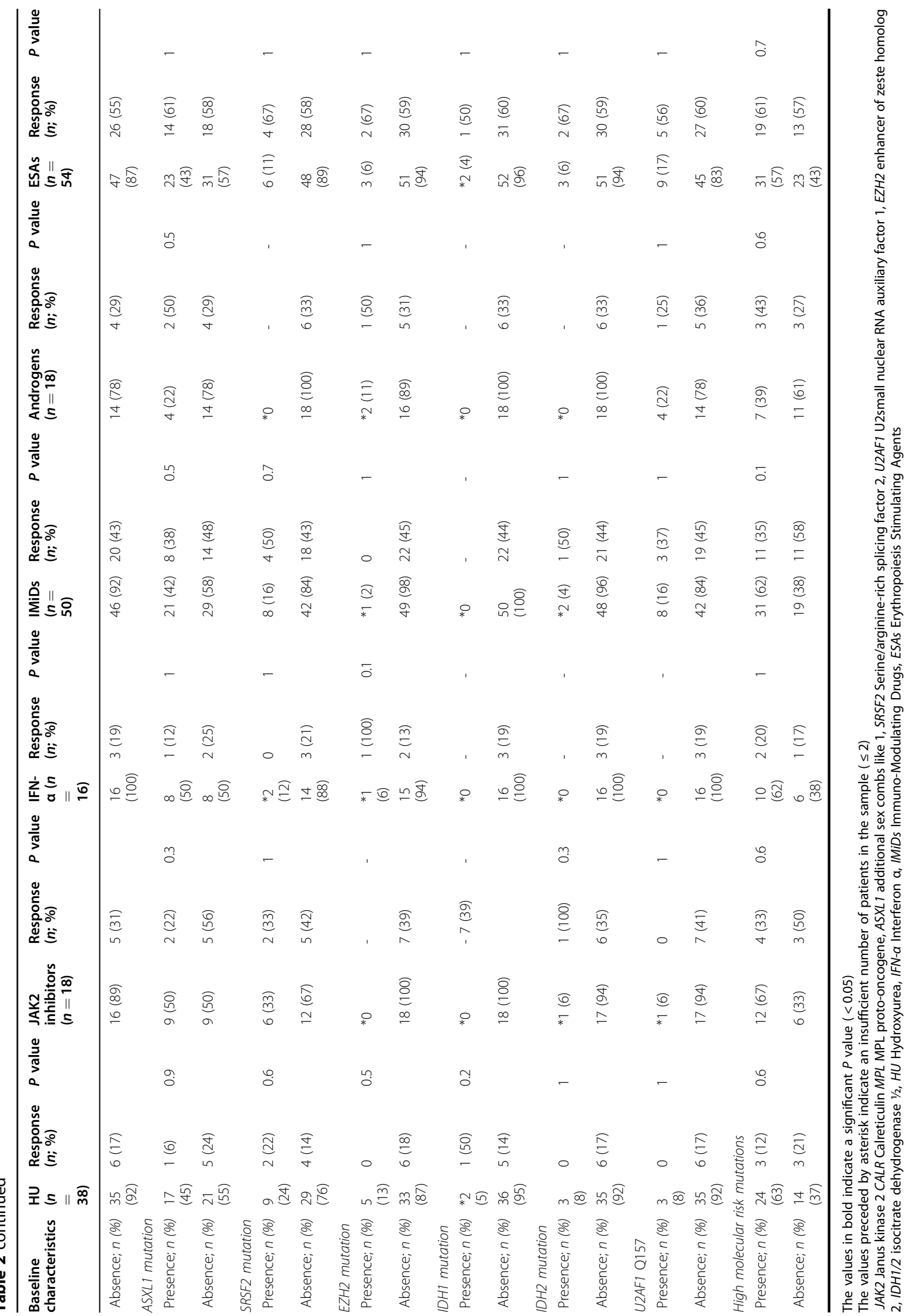


moderate to severe anemia (58\%), constitutional symptoms (31\%), platelet count $<100 \times 10^{9} / 1(20 \%)$, leukocytosis $>25 \times 10^{9} / 1(14 \%)$, and $\geq 2 \%$ circulating blasts (30\%). Driver mutational status was JAK2 59\%, CALR type 1/like $20 \%$, CALR type $2 /$ like $4 \%, M P L 7 \%$, and triple-negative $10 \%$. Karyotype included very high risk (VHR) 6\%, unfavorable $18 \%$, and favorable $76 \%$, according to the recently revised system ${ }^{15}$. Sixty percent of the patients harbored high molecular risk (HMR) mutations, including ASXL1 (45\%), SRSF2 (18\%), U2AF1Q157 (10\%), EZH2 (4\%), IDH2 (4\%), and IDH1 (2\%). MIPSS70 + version 2.0 risk distribution was as follows: very high $17 \%$, high $46 \%$, intermediate $21 \%$, low $14 \%$, and very low $2 \%$.

Overall, 249 patients were evaluable for spleen response, including 218 that were treated with the specific drugs analyzed in the current study (Table 1). Anemia response was evaluated in 222 patients (118 were red cell transfusion-dependent), including 194 (105 transfusion-dependent) that were treated with the specific drugs analyzed in the current study (Table 2).

Spleen response to $\mathrm{HU}$ was more likely in the presence of $A S X L 1$ ( $40 \%$ vs $17 \% ; p=0.06$ ) or $S R S F 2$ ( $47 \%$ vs $22 \%$; $p$ $=0.1$ ) mutations, while none of eight patients with either U2AF1Q157 or IDH2 mutations responded, none of five patients with VHR karyotype, and only one (9\%) of 11 patients with platelet count $<100 \times 10^{9} / 1$ responded. Anemia responses to $\mathrm{HU}$ were infrequent. Overall response rate to $\mathrm{HU}$ was predicted by the absence of $U 2 A F 1 \mathrm{Q} 157$ mutations (64\% vs $0 \%$; $p=0.007$ ).

Spleen response to JAK2 inhibitors was more likely in female patients ( $87 \%$ vs $50 \% ; p=0.01)$, absence of triplenegative mutational status ( $72 \%$ vs $0 \% ; p=0.01)$, and presence of CALR type $1 /$ like mutations ( $90 \%$ vs $57 \%$; $p=$ 0.06). ASXL1 ( $65 \%$ vs $65 \% ; p=1.0$ ) or SRSF2 ( $54 \%$ vs $70 \%$; $p=0.4$ ) mutations did not influence spleen response to JAK2 inhibitors. Anemia responses to JAK2 inhibitors were largely unpredictable. In order to further verify the aforementioned-observed associations, we accessed data from a previous formal clinical trial of momelotinib (JAK2 inhibitor). Data from this trial showed that among 91 evaluable patients, spleen response was higher in the presence of $C A L R$ mutations ( $73 \%$ vs $37 \% ; p=0.009)$ and female sex ( $49 \%$ vs $39 \%, p=\mathrm{NS})$.

Although significant differences were not apparent, spleen response to IFN- $\alpha$ was unlikely in the presence of $\geq 2 \%$ circulating blasts (none of six patients responded) or presence of $A S X L 1$ mutations (none of seven patients responded). IFN- $\alpha$ was often ineffective for the treatment of anemia. Spleen responses to treatment with IMiDs, androgens, or ESAs were unusual, while anemia response to all three agents was not predicted by either genetic or clinical markers.

Our observations, which require additional examination in a prospective setting, show a limited value of genetic and clinical markers in predicting response to currently available drugs for PMF (consistent with the non-specific mechanism of action for these drugs). The study also confirmed the possibility that CALR mutations and female sex predict favorable spleen response to JAK2 inhibitors and suggest $U 2 A F 1 \mathrm{Q} 157$ mutations predict unfavorable spleen response to $\mathrm{HU}$, as previously proposed in formal clinical trials.

\begin{abstract}
Author details
${ }^{1}$ Divisions of Hematology, Departments of Internal Medicine and Laboratory Medicine, Mayo Clinic, Rochester, MN, USA. 'Divisions of Hematopathology, Departments of Internal Medicine and Laboratory Medicine, Mayo Clinic, Rochester, MN, USA. ${ }^{3}$ Divisions of Laboratory Genetics and Genomics, Departments of Internal Medicine and Laboratory Medicine, Mayo Clinic, Rochester, MN, USA
\end{abstract}

\section{Competing interests}

The authors declare no competing interests.

\section{Publisher's note}

Springer Nature remains neutral with regard to jurisdictional claims in published maps and institutional affiliations.

Supplementary Information accompanies this paper at (https://doi.org/ 10.1038/s41408-018-0158-4).

Received: 14 September 2018 Accepted: 29 October 2018

Published online: 19 November 2018

\section{References}

1. Barbui, T. et al. The 2016 WHO classification and diagnostic criteria for myeloproliferative neoplasms: document summary and in-depth discussion. Blood Cancer J. 8, 15-25 (2018).

2. Tefferi, A. et al. Driver mutations and prognosis in primary myelofibrosis: MayoCareggi MPN alliance study of 1,095 patients. Am. J. Hematol. 93, 348-355 (2018).

3. Tefferi, A. et al. Targeted deep sequencing in primary myelofibrosis. Blood Adv. 1, 105-111 (2016)

4. Tefferi, A. et al. Long-term survival and blast transformation in molecularly annotated essential thrombocythemia, polycythemia vera, and myelofibrosis. Blood 124, 2507-2513 (2014).

5. Mesa, R. A. et al. The burden of fatigue and quality of life in myeloproliferative disorders (MPDs): an international Internet-based survey of 1179 MPD patients. Cancer 109, 68-76 (2007).

6. Tefferi, A. et al. Allogeneic hematopoietic stem cell transplant overcomes the adverse survival effect of very high risk and unfavorable karyotype in myelofibrosis. Am. J. Hematol. 93, 649-654 (2018).

7. Samuelson, B. et al. Hematopoietic cell transplantation for myelofibrosis: the dynamic International Prognostic Scoring System plus risk predicts posttransplant outcomes. Biol. Blood Marrow Transplant. 24, 386-392 (2018).

8. Tefferi, A. Primary myelofibrosis: 2013 update on diagnosis, risk-stratification, and management. Am. J. Hematol. 88, 141-150 (2013).

9. Vardiman, J. W. et al. The 2008 revision of the World health Organization (WHO) classification of myeloid neoplasms and acute leukemia: rationale and important changes. Blood 114, 937-951 (2009).

10. Arber, D. et al. The 2016 revision to the World Health Organization classification of myeloid neoplasms and acute leukemia. Blood 127, 2391-2405 (2016).

11. Guglielmelli, P. et al. MIPSS70: mutation-enhanced international prognostic score system for transplantation-age patients with primary myelofibrosis. J. Clin. Oncol. 36, 310-318 (2018).

12. Tefferi, A. et al. MIPSS70+Version 2.0: mutation and karyotype-enhanced international prognostic scoring system for primary myelofibrosis. J. Clin. Oncol. 36, 1769-1770 (2018). 
13. Tefferi, A. et al. GIPSS: genetically inspired prognostic scoring system for primary myelofibrosis. Leukemia 32, 1631-1642 (2018).

14. Tefferi, A. et al. Revised response criteria for myelofibrosis: International Working Group-Myeloproliferative Neoplasms Research and Treatment (IWG-
MRT) and European LeukemiaNet (ELN) consensus report. Blood 122, 1395-1398 (2013).

15. Tefferi, A. et al. Revised cytogenetic risk stratification in primary myelofibrosis: analysis based on 1002 informative patients. Leukemia 32, 1189-1199 (2018). 